\title{
Storage of Light in Atomic Vapor
}

\author{
D. F. Phillips, A. Fleischhauer, A. Mair, and R. L. Walsworth \\ Harvard-Smithsonian Center for Astrophysics, Cambridge, Massachusetts 02138
}

\author{
M. D. Lukin \\ ITAMP, Harvard-Smithsonian Center for Astrophysics, Cambridge, Massachusetts 02138
}

(Received 22 December 2000)

\begin{abstract}
We report an experiment in which a light pulse is effectively decelerated and trapped in a vapor of $\mathrm{Rb}$ atoms, stored for a controlled period of time, and then released on demand. We accomplish this "storage of light" by dynamically reducing the group velocity of the light pulse to zero, so that the coherent excitation of the light is reversibly mapped into a Zeeman (spin) coherence of the Rb vapor.
\end{abstract}

DOI: $10.1103 /$ PhysRevLett.86.783

Photons are the fastest and most robust carriers of information, but they are difficult to localize and store. The present Letter reports a proof-of-principle demonstration of a technique $[1,2]$ to trap, store, and release excitations carried by light pulses. Specifically, a pulse of light which is several kilometers long in free space is compressed to a length of a few centimeters and then converted into spin excitations in a vapor of $\mathrm{Rb}$ atoms. After a controllable time, the process is reversed and the atomic coherence is converted back into a light pulse.

This "light-storage" technique is based on the recently demonstrated phenomenon of ultraslow light group velocity [3], which is made possible by electromagnetically induced transparency (EIT) [4]. In "slow-light" experiments an external optical field (the "control field") is used to make an otherwise opaque medium become transparent near an atomic resonance. A weak optical field (the "signal field") at a particular frequency and polarization can then propagate without dissipation and loss but with a substantially reduced group velocity. Associated with slow light is a considerable spatial compression, which allows a signal pulse to be almost completely localized in the atomic medium. In addition, as the signal light propagates the atoms are driven into a coherent superposition of (typically) Zeeman or hyperfine states that is strongly coupled to the light via a Raman transition. The coupled light and atomic excitations can be efficiently described as a single form of dressed-state excitation known as the "dark-state polariton" [2]. In order to store a light pulse, we smoothly turn off the control field, which causes the dark-state polariton to be adiabatically converted into a purely atomic excitation (a Rb Zeeman coherence in the experiment reported here) which is confined to the vapor cell. Turning the control field back on reverses the process: the darkstate polariton is adiabatically restored to an optical excitation, which can then leave the sample cell.

A key feature of the light-storage method is its nondestructive nature [5]. Specifically, the atomic spin excitations do not couple to electronic excited states and are thus immune to spontaneous emission. Therefore, in the ideal limit, the light-storage process is completely coherent, i.e.,
PACS numbers: 42.50.Gy, 03.67.-a

it is expected that the phase and quantum state of the signal pulse can be preserved. In practice the storage time is limited by the atomic coherence lifetime. In the present experiment we were able to measure storage times up to $\sim 0.5 \mathrm{~ms}$. The nondestructive nature of the light-storage technique makes it an attractive candidate for potential applications involving coherent communication between distant quantum-mechanical systems [1].

Before proceeding we note that adiabatic passage has also been considered for photon state storage in single atoms in the context of cavity QED [6] and that encouraging experimental progress in this direction has recently been reported [7]. In contrast, the light-storage technique demonstrated here involves collective atomic excitations in an optically dense, many-atom system which is considerably more robust and reliable than the single-atom approach and is thus much easier to implement experimentally. Efficient protocols for quantum computation [8], teleportation [9], and squeezing [10] using such excitations are currently being actively investigated. Other related work includes studies of the "stopping" of light in moving media [11] and investigations of efficient nonlinear optical effects using atomic coherences [12].

The present experiment can be understood qualitatively by considering a $\Lambda$ configuration of atomic states coupled by a pair of optical fields (see Fig. 1a). Here the control field (Rabi frequency $\Omega_{c}$ ) and signal field $\left(\Omega_{s}\right)$ are represented, respectively, by right and left circularly polarized light $\left(\sigma_{+}\right.$and $\left.\sigma_{-}\right)$derived from a single laser beam. These light fields couple pairs of Zeeman sublevels of electronic ground state $\left(5^{2} S_{1 / 2}\right) \mathrm{Rb}$ atoms $(|-\rangle,|+\rangle)$, with magnetic quantum numbers differing by two, via the excited $5^{2} P_{1 / 2}$ state [13].

We performed light-storage experiments in atomic $\mathrm{Rb}$ vapor at temperatures $\sim 70-90^{\circ} \mathrm{C}$, which corresponds to atomic densities $\sim 10^{11}-10^{12} \mathrm{~cm}^{-3}$. Under these conditions the 4-cm-long sample cell was normally completely opaque for a weak optical field near the $\mathrm{Rb} D_{1}$ resonance $(\simeq 795 \mathrm{~nm})$. We derived the control and signal beams from the output of an extended cavity diode laser by carefully controlling the light polarization as shown in the 
a)

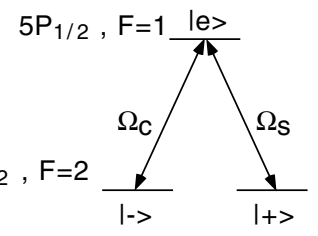

b)

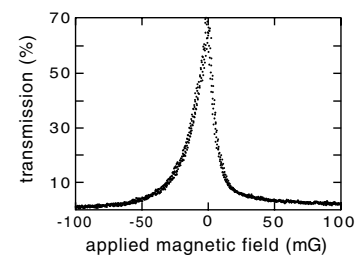

c)

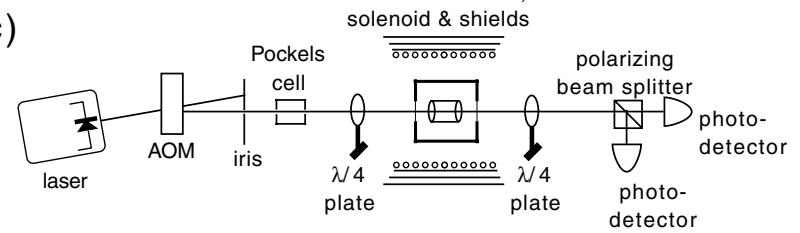

FIG. 1. (a) $\Lambda$-type configuration of ${ }^{87} \mathrm{Rb}$ atomic states resonantly coupled to a control field $\left(\Omega_{c}\right)$ and a signal field $\left(\Omega_{s}\right)$. (b) A typical observed Rb Faraday resonance in which the transmission intensity is shown as a function of magnetic field (i.e., detuning of Zeeman levels from the two-photon resonance condition). The full width of this resonance is about $30 \mathrm{mG}$ which corresponds to a $40 \mathrm{kHz}$ level shift. (c) Schematic of the experimental setup.

experimental schematic in Fig. 1c. For the data presented here we employed the $5^{2} S_{1 / 2}, F=2 \rightarrow 5^{2} P_{1 / 2}, F=1$ transition in ${ }^{87} \mathrm{Rb}$. The control field was always much stronger than the signal field $\left(\Omega_{c} \gg \Omega_{s}\right)$; hence, most of the relevant atoms were in the $5^{2} S_{1 / 2}, F=2, M_{F}=+2$ magnetic sublevel. In this case the states $|-\rangle,|+\rangle$ of the simplified three-level model correspond, respectively, to $\left|F=2, M_{F}=0\right\rangle$ and $\left|F=2, M_{F}=+2\right\rangle$. The input laser beam was collimated and focused onto a 3-mm spot in the sample cell. By using a fast Pockels cell we slightly rotated the polarization of the input light to create a weak pulse of left circularly polarized $\left(\sigma_{-}\right)$light, which served as the signal field. Input peak powers were about $1 \mathrm{~mW}$ and $100 \mu \mathrm{W}$ for the $\sigma_{+}$and $\sigma_{-}$components. We monitored transmission of the $\sigma_{-}$light pulse using a $\lambda / 4$ wave plate and polarizing beam splitter. In order to ensure long lifetimes of the atomic Zeeman coherences, we magnetically shielded the Rb cell and filled it with about 5 torr of He buffer gas. We used a precision solenoid to control the static magnetic field along the propagation direction of the optical beam.

We first consider the case of $\mathrm{cw}$ signal and control fields. Figure $1 \mathrm{~b}$ displays a typical transmission spectrum for the signal $\left(\sigma_{-}\right)$field obtained by scanning the magnetic field and thereby changing the effective two-photon detuning. Note that due to the induced transparency the signal field transmission is maximal for zero magnetic field, even though most atoms are in the state $|+\rangle$. Outside of the transparency window (magnetic fields $>20 \mathrm{mG}$ ) the $\mathrm{Rb}$ vapor is completely opaque to $\sigma_{-}$light.

We next present a demonstration of light storage. Typical input $\sigma_{-}$signal pulses had a temporal length of $\sim 10$ to $30 \mu \mathrm{s}$, corresponding to a spatial length of several kilometers in free space. Upon entrance into the $\mathrm{Rb}$ cell the signal pulse was spatially compressed by more than 5 orders of magnitude, due to the reduction in group velocity, as estimated from the observed change in pulse propagation. In order to trap, store, and release the signal pulse, we used an acousto-optic modulator to turn off the control field smoothly over about $3 \mu$ s while much of the signal pulse was contained in the $\mathrm{Rb}$ cell. After some time interval, we turned the control field on again, thereby releasing the stored portion of the signal pulse.

An example of the observed light storage is shown in Fig. 2. Typically, two time-resolved $\sigma$ - signal pulses were registered by the photodetector in the process of light storage and release. First, a fraction of the signal pulse left the cell before the control field was turned off, which resulted in an observed signal that was not affected by the storage operation (peak I in each plot of Fig. 2). This untrapped light was delayed by about $30 \mu \mathrm{s}$ as compared to free-space propagation due to the slow group velocity $\left(v_{g} \sim 1 \mathrm{~km} / \mathrm{s}\right)$. The second observed signal pulse was
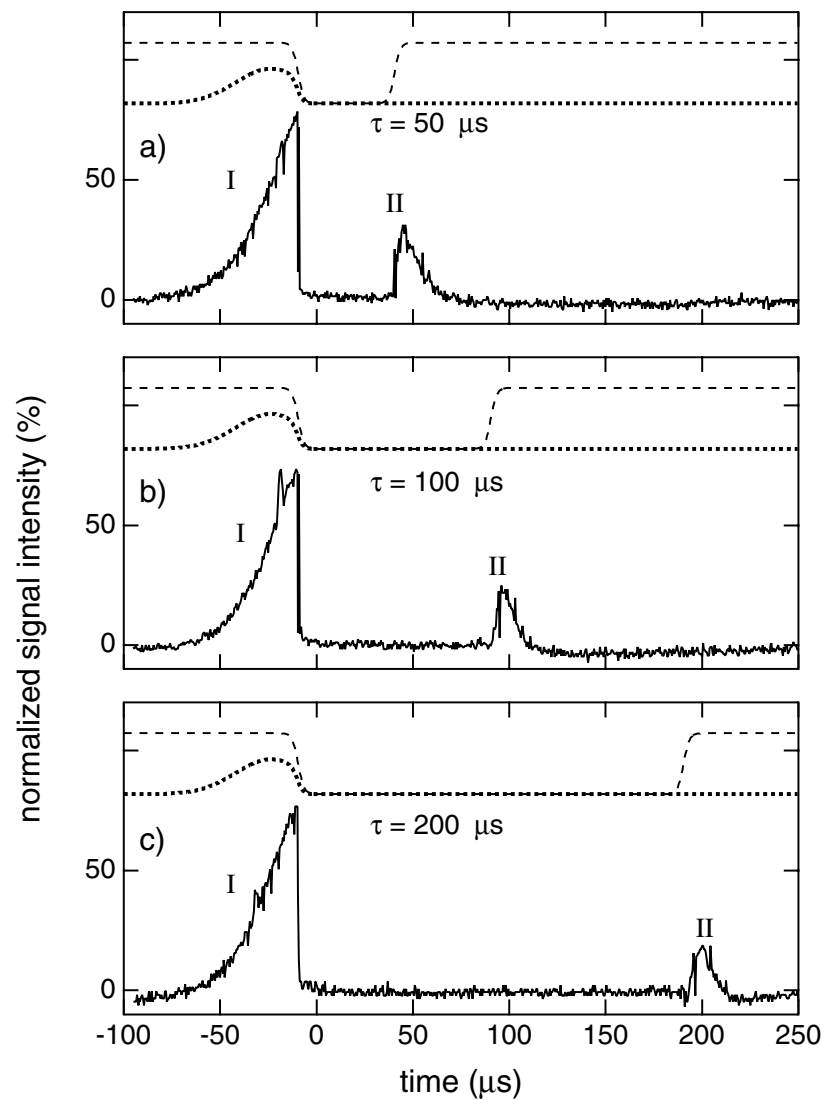

FIG. 2. Observed light pulse storage in a ${ }^{87} \mathrm{Rb}$ vapor cell. Examples are shown for storage times of (a) $50 \mu \mathrm{s}$, (b) $100 \mu \mathrm{s}$, and (c) $200 \mu \mathrm{s}$. (Background transmission from the control field, which leaks into the signal field detection optics, has been subtracted from these plots.) Signals are normalized to the peak intensity of the light transmitted through the stationary EIT medium. Shown above the data in each graph are calculated representations of the applied control field (dashed line) and input signal pulse (dotted line). Estimated peak $\Omega_{c} \sim 3 \mathrm{MHz}$, $\Omega_{s} \sim 0.9 \mathrm{MHz}$, and $\sqrt{\kappa} \sim 2000 \mathrm{MHz}$. 
light that was stored in atomic excitations for a time interval $\tau$. Note that no output signal was observed as long as the control field was off; rather, the released signal pulse was detected only after the control field was turned back on (peak II in each plot of Fig. 2). The controlled light storage is the principal result of this Letter. We observed that the amplitude of the released signal pulse decreased as $\tau$ increased. We could resolve released light pulses without signal averaging for storage intervals up to $\tau \simeq 0.5 \mathrm{~ms}$.

We turn now to a theoretical interpretation of the experimental results. We consider the propagation of a signal pulse in an EIT medium (along the $z$ direction) subject to a time-dependent control field. We assume that the signal field is always weaker than the control field and that the signal field group velocity is always much smaller than the control field group velocity. (The latter assumption allows us to neglect the retardation and spatial dependence of the control field.)

As noted above, the dynamical trapping of signal pulses can be understood in terms of dark-state polaritons [2]. These are coupled superpositions of photonic and spin wavelike excitations, defined by a transformation:

$$
\begin{gathered}
\Psi(z, t)=\cos \theta(t) \Omega_{s}(z, t)-\sin \theta(t) \sqrt{\kappa} \rho_{-+}(z, t), \\
\cos \theta(t)=\frac{\Omega_{c}(t)}{\sqrt{\Omega_{c}^{2}(t)+\kappa}}, \quad \sin \theta(t)=\frac{\sqrt{\kappa}}{\sqrt{\Omega_{c}^{2}(t)+\kappa}} .
\end{gathered}
$$

Here $\rho_{-+}$is the atomic coherence between states $|-\rangle$and $|+\rangle$. Also, $\kappa=3 n \lambda^{2} \gamma_{r} c / 8 \pi$, where $n$ is the ${ }^{87} \mathrm{Rb}$ density, $\lambda$ is the wavelength and $\gamma_{r}$ is the natural linewidth of the $D_{1}$ transition, and $c$ is the free-space speed of light.

In the ideal limit, corresponding to vanishing dephasing of the atomic coherence and perfect adiabatic following, the polariton propagation is described by [2]

$$
\Psi(z, t)=\Psi\left[z-\int_{t_{0}}^{t} d t^{\prime} v_{g}\left(t^{\prime}\right), t=t_{0}\right],
$$

where the time-dependent group velocity is

$$
v_{g}(t)=c \times \cos ^{2}(\theta)=c \times \frac{\Omega_{c}^{2}(t)}{\Omega_{c}^{2}(t)+\kappa} .
$$

In the stationary case, $v_{g}(t)=v_{g}^{0}$, and after initial pulse compression at the entrance of the cell, the polariton describes the well-known EIT-like propagation of coupled light and atomic coherence. Remarkably, however, when the intensity of the control field is changed during the pulse's propagation through the atomic medium, the polariton can preserve its shape, amplitude, and spatial length, while its group velocity and the ratio of the light and matter components are altered. In particular, when the group velocity is reduced to zero by turning off the control beam, the polariton becomes purely atomic $(\cos \theta=0)$ and its propagation is stopped. The state of the input light pulse is thereby mapped into the atomic coherence $\rho_{-+}$. The coherence stored within the cell $\left(0<z<L_{\text {cell }}\right)$ after switching off the control field over the time interval

$$
\begin{aligned}
& {\left[t_{0}, t_{1}\right] \text { is given by }} \\
& \rho_{-+}\left(z, t_{1}\right)=-\sqrt{\frac{c}{v_{g}^{0} \kappa}} \Omega_{s}\left(z=0, \frac{\int_{t_{0}}^{t_{1}} v_{g}\left(t^{\prime}\right) d t^{\prime}-z}{v_{g}^{0}}\right) .
\end{aligned}
$$

If the control beam is turned back on after a storage interval $\tau$, the polariton is accelerated and the atomic coherence $\rho_{-+}$is mapped back into light. The released light pulse has a shape, amplitude, and spatial length proportional to the coherence after the storage interval:

$\Omega_{s}(z, t)=-\cos \theta(t) \sqrt{\kappa} \rho_{-+}\left(L_{\text {cell }}-\int_{t_{2}}^{t} v_{g}\left(t^{\prime}\right) d t^{\prime}, t_{2}\right)$,

where $t_{2}=t_{1}+\tau$. In principle, complete storage and retrieval of the input light pulse is possible [14]. To make a comparison with experimental results, however, deviations from this ideal limit must be considered: e.g., decay of the Zeeman coherence and nonadiabatic corrections. Results of theoretical calculations for the conditions of our experiment (Fig. 3) are in good agreement with the measurements displayed in Fig. 2. Under realistic conditions, the light-storage time is always limited by loss of atomic coherence (Figs. 2 and 3). In the present experiment, for example, the $\mathrm{Rb}$ atoms slowly escape from the region of interaction with the light beam; at high atom densities, spin coherences decay due to spin-exchange collisions.

It is also important to consider the assumption of adiabatic following and its effect on the dynamic method for

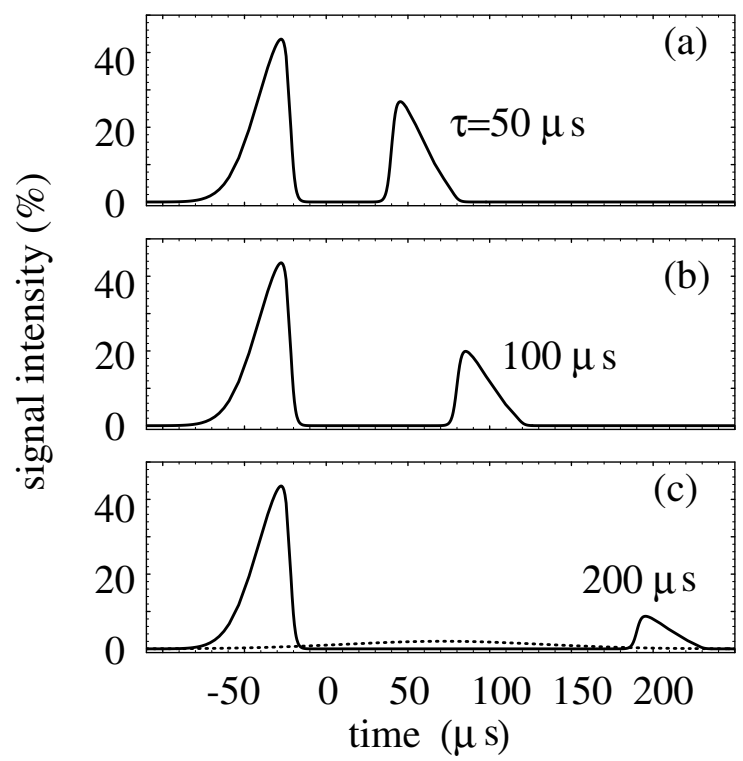

FIG. 3. Theoretical simulations of light storage in a three-state $\Lambda$ system. Solid curves in each graph correspond to the experimental conditions of Figs. 2a-2c. Dephasing of the coherence is modeled by an exponential with a decay constant of $150 \mu \mathrm{s}$. The dotted line in Fig. 3c corresponds to the usual EIT propagation with a cw control field of 5 times weaker intensity than the input value of the dynamic case. 
group velocity reduction, as compared to the conventional, stationary approach based on EIT. Naively, Eq. (3) indicates that long light pulse delays can be obtained by simply using a cw control field of sufficiently low intensity. However, for pulses similar to those used in the dynamic trapping method we failed to observe long delays $(\sim 100 \mu \mathrm{s})$ using the stationary EIT technique. This failure is due to the breakdown of adiabatic following.

The essence of adiabatic following is that the light pulse spectrum should be contained within a relatively narrow transparency window $\Delta \omega$ (Fig. 1b) to avoid loss and dissipation. The magnitude of $\Delta \omega$ is determined by both the control field intensity and the opacity of the atomic medium [15]. In conventional EIT propagation, a weaker control field induces a narrower transmission spectrum. For a fixed bandwidth of the propagating signal pulse, such spectral narrowing causes absorption of certain pulse spectral components and inevitably destroys propagating light. This loss is consistent with our experimental observations using the stationary EIT technique and is confirmed by our calculations (e.g., the dotted line in Fig. 3c), which include nonadiabatic corrections corresponding to a finite width of the EIT spectrum.

The observed light pulses stored and released by $d y$ namic reduction of the group velocity are obviously not destroyed in spite of the narrowing of the transparency window. This important result is in agreement with the theoretical predictions of Ref. [2], where it was pointed out that adiabatic following occurs as long as the product of the propagation distance and the normal opacity of the medium [i.e., the absorption length $l_{\mathrm{abs}}=\kappa /\left(\gamma_{\mathrm{opt}} c\right)$, where $\gamma_{\mathrm{opt}}$ is the total linewidth of the optical transition] is smaller than the square of the spatial light pulse length in the medium. In other words, adiabaticity can be preserved with the dynamic light-storage method as long as the input pulse bandwidth is within the initial transparency window. A remarkable feature of the dark-state polariton is that its spatial length remains unchanged in the process of deceleration. Hence, a dynamic reduction in group velocity is accompanied by a narrowing of the polariton frequency spectrum (bandwidth). In this case, adiabatic following occurs even when the group velocity is reduced to zero, which is in good agreement with the experimental results presented here.

In conclusion, we have demonstrated that it is possible to control the propagation of light pulses in optically thick media by dynamically changing the group velocity. In particular, a light pulse can be trapped and stored in an atomic coherence; after a controllable delay this coherence can be converted back into a light pulse.
It is a pleasure to thank M. Fleischhauer and S. Yelin for many fruitful ideas and collaboration on theoretical aspects of this work. We also thank S. Harris and M. O. Scully for many stimulating discussions. This work was partially supported by the National Science Foundation and the Office of Naval Research.

Note added.-Light storage has also been observed recently in ultracold atomic gas [16], with storage times of the same order of magnitude as those reported here.

[1] M. D. Lukin, S. F. Yelin, and M. Fleischhauer, Phys. Rev. Lett. 84, 4232 (2000); L. M. Duan, J. I. Cirac, and P. Zoller (unpublished).

[2] M. Fleischhauer and M. D. Lukin, Phys. Rev. Lett. 84, 5094 (2000).

[3] L. V. Hau, S.E. Harris, Z. Dutton, and C.H. Behroozi, Nature (London) 397, 594 (1999); M. Kash et al., Phys. Rev. Lett. 82, 5229 (1999); D. Budker et al., ibid. 83, 1767 (1999).

[4] See, e.g., S. E. Harris, Phys. Today 50, No. 7, 36 (1997).

[5] Dissipative techniques for the partial transfer of quantum statistics from light to atoms are reported in A. Kuzmich, K. Mølmer, and E. S. Polzik, Phys. Rev. Lett. 79, 4782 (1997); J. Hald, J. L. Sørensen, C. Schori, and E. S. Polzik, Phys. Rev. Lett. 83, 1319 (1999).

[6] J. I. Cirac, P. Zoller, H. J. Kimble, and H. Mabuchi, Phys. Rev. Lett. 78, 3221 (1997).

[7] M. Hennrich, T. Legero, A. Kuhn, and G. Rempe, Phys. Rev. Lett. 85, 4872 (2000).

[8] M. D. Lukin et al., quant-ph/0011028.

[9] L. Duan, J. I. Cirac, P. Zoller, and E. Polzik, quant-ph/ 0003111.

[10] A. Kuzmich, L. Mandel, and N. Bigelow, Phys. Rev. Lett. 85, 1594 (2000).

[11] O. Kocharovskaya, Yu. Rostovtsev, and M. O. Scully, Phys. Rev. Lett. 86, 628 (2001).

[12] H. Schmidt and A. Imamoğlu, Opt. Lett. 21, 1936 (1996); S. E. Harris and Y. Yamamoto, Phys. Rev. Lett. 81, 3611 (1998); S. E. Harris and L. V. Hau, ibid. 82, 4611 (1999); M. D. Lukin and A. Imamoğlu, ibid. 84, 1419 (2000).

[13] For observation of Zeeman-coherence-based EIT in a dense medium, see V. A. Sautenkov et al., Phys. Rev. A 62, 023810 (2000).

[14] In our present experiment up to $\sim 50 \%$ of the input light excitation has been trapped. We anticipate that the stored fraction can be increased by either using a larger densitylength product or with an optical cavity [1].

[15] S. E. Harris, Phys. Rev. Lett. 70, 552 (1993); M. D. Lukin et al., Phys. Rev. Lett. 79, 2959 (1997).

[16] C. Liu, Z. Dutton, C. H. Behroozi, and L. V. Hau, Nature (London) (to be published). 